\title{
A Bayesian-Rational Framework for Conspiracy Theories
}

\author{
Gabriel Doyle (gdoyle@sdsu.edu) \\ Department of Linguistics \\ Asian/Middle Eastern Languages \\ Digital Humanities Initiative \\ San Diego State University
}

\begin{abstract}
In our present era of fractured politics, social media, and fake news, conspiracy theories are as prominent as ever. While conspiracy theories are often dismissed as pathological or irrational reasoning, belief in at least some conspiracy theories could arise from a Bayesian rational system that is merely wrong, rather than truly irrational. This paper lays out a framework for understanding how conspiracy theories could arise from rational Bayesian cognition, identifying four potential sources for conspiracy theory belief in a primarily rational framework: elevated prior belief in CTs, different likelihoods, missing non-conspiratorial explanations, and non-epistemic utilities.
\end{abstract}

Keywords: Bayesian modelling; decision making; belief formation; conspiracy theories; rationality

\section{Introduction}

Conspiracy theories seem obviously irrational. Many are fanciful stories that strain credulity, or assume hundreds of collaborators are operating in unerring synchronicity. The very term "conspiracy theory" conveys disdain for an idea, and everyone from politicians to internet commenters know that labelling something a conspiracy theory is to reject it out of hand, an act of "rhetorical violence" (Wood, 2016). For instance, when reporters asked New Jersey governor Chris Christie if there were political motivations behind lane closures on one of the major bridges leading from New Jersey to New York City, his office rejected the notion as a "wild-eyed conspiracy theory" unworthy of comment (Benen, 2014). How could a rational person believe in complex shadowy forces exerting secret influences to change the state of the world to their benefit?

Well, for one, it turns out that some conspiracies are real. After a few months of investigation, two associates of Christie were convicted of conspiracy for engineering the closure as political payback, precisely the theory that Christie had dismissed (Racioppi, 2016). So we must ask: what distinguishes irrational belief in a conspiracy theory from a rational belief in an as-yet-unexposed actual conspiracy? Perhaps there is a grain of rationality underlying belief in some-though not necessarily all-conspiracy theories. If so, this could have significant impacts in understanding how to deal with the spread of conspiracy theories, especially dangerous contemporary ones like climate change denial, anti-vaccination, and QAnon.

This paper lays out a framework for a rational underpinning for conspiracy theories (CTs), which may be augmented by irrational/non-normative additions as needed. We build on research showing that seemingly-irrational belief polar- ization, where two people encountering the same information update their beliefs in opposite directions, can arise from Bayesian-rational frameworks (Cook \& Lewandowsky, 2016; Olsson, 2020). We develop a basic Bayesian framework for considering conspiratorial and non-conspiratorial explanations for phenomena, and identify four ways that normatively optimal reasoning could lead someone to support a CT: elevated prior beliefs in conspiracies, likelihood function differences, missing hypotheses, and alternative utility functions. We consider how to test this framework and the implications of rationality in CTs for interventions.

\section{Background}

\section{Conspiracy theories: then and now}

While CTs are often thought of as a fairly new phenomenon, conspiracy theorizing has a long history. Proto-CTs, if not full-blown CTs, date back to the Roman Empire, such as rumors about the death of Emperor Nero in $68 \mathrm{CE}$ (Pagán, 2008). A wide range of antisemitic conspiracy theories have percolated through from the Middle Ages to the present day, including the "blood libel" and the Protocols of the Elders of Zion. Conspiracy theories aided the witch hunts and moral panics of these times as well, motivated by belief in supernatural conspiracies (Groh, 1987). In more recent American history, the canonical CTs come from the 1960s, most notably the John F. Kennedy assassination and the Moon landing. Since then, belief in CTs has been helped along by the discovery of confirmed conspiracies in American politics, such as the Watergate break-in, the Iran-Contra affair, and numerous small-scale conspiracies like the New Jersey bridge closure. Recent years have seen a further explosion in CTs, such as climate change denialism, chemtrails, and the CT of the moment, QAnon.

This historical ubiquity of CTs may be due in part to the range of utilities that they can provide to believers. Some provide a reassuring sense of order in the face of otherwise arbitrary events (Barkun, 2003), a significant enough motivation that some researchers include it in their definition of a CT (Keeley, 1999). Believing in a CT can provide a satisfying sense of understanding hidden "counterknowledge" (Gosa, 2011), or being part of a cooperative narrative building experience that provides a sense of community (Franks, Bangerter, Bauer, Hall, \& Noort, 2017). CT believers can develop rich narratives that become intimidatingly complex to an outsider and reassuringly complete to believers (Wood, Douglas, \& Sutton, 2012; Raab, Ortlieb, Auer, Guthmann, \& Carbon, 2013). 
Conspiracy theories may seem unworthy of serious study, easy to dismiss as mere irrationality or fringe beliefs with little impact on cognitive science or the world at large. But conspiracy theorizing couples with general cognition in ways that make it more than a frivolous concern. Douglas and Sutton (2008) show that exposure to information from a CT can influence people's beliefs about the topic of the CT without them being aware of the shift. Endorsement of CTs is also associated with negative cognitive behaviors, such as increased racism and feelings of powerlessness (Swami, 2012; Jolley \& Douglas, 2014). CT belief can lead people to engage in riskier behaviors due to a denial of real dangers (Hoyt et al., 2012), an especially important feature to address at present with the need for action to prevent climate change and to encourage COVID-19 vaccinations. In addition, there is substantial evidence that belief in one CT correlates with belief in others (Brotherton, French, \& Pickering, 2013), leading to potential snowball effects.

Making matters worse, experimental evidence argues that labelling an idea as a "conspiracy theory" may not dissuade people from adopting it (Wood, 2016). Understanding what leads people to believe in CTs is critical to developing effective interventions (Swami et al., 2013) and inoculations (van der Linden, Leiserowitz, Rosenthal, \& Maibach, 2017) against CTs. Distinguishing between rational and irrational aspects of CT belief can help with the design of better interventions, by separating beliefs reached through rational analysis with incorrect priors/likelihoods from beliefs reached through irrational aspects such as confirmation biases or alternative utilities. It may also help determine whether there are identifiable differences between unreasonable CTs and reasonable skeptical beliefs (Huneman \& Vorms, 2018).

\section{Defining conspiracy theories}

Despite the long history of conspiracy theorizing, until recently, there has been limited academic study on CTs, and the recent work has been spread across a variety of disciplines (Goreis \& Voracek, 2019). Conspiracy theories span such a wide range of topics that even creating a definition for the term is difficult, and different researchers have developed a range of definitions; Huneman and Vorms (2018) argue that a single unified account of conspiracy theories is impossible, and the line between unreasonable CTs and reasonable skepticism is blurry. As such, this paper will be intentionally inclusive in its definition of a conspiracy theory. Some beliefs that meet this definition may not fit the rational framework developed here, and some may be mainstream enough to avoid the CT label. Our hope in being expansive is to capture most CTs and as many related phenomena as possible under this explanatory framework.

Based on Brotherton and French (2014)'s definition, we say that a CT is any explanation for observed data/behaviors that a) relies on a person or group exerting hidden influences to achieve their goals and generate the observed data, and b) is considered implausible and/or unjustified by most people.
Point A separates CTs from ideas that are non-conspiratorial, while point B separates CTs in the pejorative sense from established or plausible conspiracies, which would be unlikely to be considered irrational. Most prominent CTs will fit this definition, especially the climate change denial and Satanic child trafficking CTs that we will analyze within the proposed framework.

\section{Evidence for rationality in CT belief}

Perhaps the biggest open question in conspiracy theory research is whether the processes by which people adopt CTs are inherently irrational or simply incorrect applications of rational frameworks. While many common CTs are extreme and absurd, others are not far off from observed facts. The difference between these CTs and rational skeptical beliefs may be, over a historical timeframe, whether or not the conspiracy turns out to be true. Many historical events have involved conspiracies, including assassinations (Julius Caesar, etc.), price-fixing, match-fixing, medical experimentation (e.g., the Tuskegee Syphilis Study), suppression of scientific results (e.g., Big Tobacco), and other common CT topics. For some of these, contemporary belief in what was later revealed to be the actual conspiracy would have been derided as conspiracy theorizing. Simply put, some (though relatively few) CTs do end up being approximations of the truth. This suggests that there is an underlying kernel of rationality to some CTs, and this is backed up by some psychological studies into CT belief and related behaviors.

Other seemingly irrational behaviors have been explained as normative outcomes of complex Bayesian rational cognition, such as confirmation biases (Navarro \& Perfors, 2011) and jumping to conclusions (Vul, Goodman, Griffiths, \& Tenenbaum, 2014). A particularly relevant case for CTs comes from studies of belief polarization-in which different people exposed to the same data may diverge in the conclusions they draw and become more entrenched in their contrasting prior beliefs. Jern, Chang, and Kemp (2014) showed that this can arise normatively when two people have different likelihood functions, due to a hidden variable that influences the conditional probability of the data under each hypothesis.

Cook and Lewandowsky (2016) applied this to belief in, or denial of, climate change. Participants shown the same statement of scientific consensus about climate change diverged in their posterior beliefs, with each side becoming more confident in their prior preference. They argue that this polarization is normative because the likelihood of climate change being real depends not only on the obvious connection between evidence and reality but also on a hidden factor of trust in the scientists who compose the consensus. Olsson (2020) pushes this idea of differential trust leading to polarization further by showing that in a trust-driven Bayesian agent-based model, polarized beliefs (e.g., a CT and non-CT explanation for observed data) is a natural outcome, especially if holders of the two belief systems trust different information sources.

Signals of rationality have been found in studies of CTs 
specifically dating back at least to McCauley and Jacques (1979), who showed that participants' posterior beliefs in a conspiratorial explanation for a presidential assassination were not significantly biased from the normative estimates based on their self-reported likelihood and prior terms. Furthermore, their participants showed stronger belief in conspiracies for successful assassinations over unsuccessful attempts, corresponding to the participants' belief in higher likelihoods of success with a conspiracy than a lone assassin. Madsen, Bailey, and Pilditch (2018) demonstrate that rational agents communicating in large networks can create CTsupporting echo chambers, with larger networks increasing the probability of such echo chambers. Huneman and Vorms (2018) argue that CTs become rational as trust decreases and the rate of conspiratorial behaviors rise, as under authoritarian governments. However, no complete Bayesian framework for explaining CTs has yet been proposed.

It is worth noting that irrational explanations have also been developed for belief polarization and CTs, relying on heuristics that merely approximate rationality (Zimper \& Ludwig, 2009), or on cognitive failures that bring in nonoptimal information (Taber \& Lodge, 2006). In proposing a rational framework for CTs, we do not assume that all CTs come from such a rational framework, only that a meaningful proportion of them are at least partially based on rational analysis. Further study can develop this framework to identify rational components within CTs, as well as deviations from rationality.

\section{A rational framework for conspiracy theories}

This section lays out a basic Bayesian rational framework by which a person seeks the most satisfying explanation $H$ for a set of observed phenomena $D$. Posterior probabilities of $\mathrm{CT}$ and non-CT hypotheses $\left(H_{C T}, H_{N C}\right)$ are calculated in proportion to the product of the likelihoods and priors for each hypothesis, following Bayes' Rule. The epistemic utility of choosing a high-posterior hypothesis is combined with additional utilities derived from the hypotheses themselves to determine which hypothesis is preferred. We will see that there are at least four ways that such a framework could prefer a CT, even before adding any significant deviations from rationality. With this framework in place, future experiments can measure or manipulate the various inputs to this model to determine if the changes in a participant's belief indeed follow the rational framework's predictions, similar to Cook and Lewandowsky (2016)'s experiments on belief polarization.

For simplicity's sake in the basic framework, we will suppose that there are two primary explanations available: $H_{C T}$, the conspiratorial hypothesis, and $H_{N C}$, the non-CT hypothesis that the data is explained by some simple and nonnefarious means or simple coincidence. (Later, we will also consider a third hypothesis $H_{T}$ for cases where the true explanation is complex but still not conspiratorial.) Using Bayes' Rule, the posterior probability of each of these explanations, after observing data $D$, is proportional to the likelihood of seeing this data under a given hypothesized source $H$ times the prior belief in that source:

$$
p(H \mid D) \propto p(D \mid H) p(H)
$$

A rational decision-maker seeks to maximize the utility of their decision. In this framework, this utility is primarily derived by identifying the true source of the data, which we can call the epistemic utility. The epistemic utility is:

$$
U_{\text {epistem }}(H ; D)=\log p(H \mid D) \propto \log p(D \mid H)+\log p(H)
$$

However, other utilities may factor into the decisionmaking, such as personal satisfaction from a certain belief or the notion that awareness of a conspiracy may help the believer avert the conspirators' preferred (negative) outcome. As a result, the utility of each hypothesis is the combination of the epistemic and any non-epistemic utilities:

$$
\begin{aligned}
U(H \mid D) & =U_{\text {epistem }}(H \mid D)+U_{\text {other }}(H) \\
& \propto \log p(H \mid D)+\log p(H)+U_{\text {other }}(H)
\end{aligned}
$$

Note that CTs (and even some non-CT explanations) are notoriously vague. While in some cases, the hypotheses can be made explicit, with specific mechanisms connecting the data and hypotheses, in others they may be vague or represent a whole class of related hypothesis. For instance, in many UFO sightings, there is no single convincing non-alien explanation for the observed phenomenon, yet most people believe that some unspecified non-alien explanation is more believable than any alien explanation. Similarly, many CTs are incomplete or contain contradictory ideas (Wood et al., 2012). Depending on the whether the hypotheses are explicit or vague notions that span multiple explicit hypotheses, we can do these calculations for the specific hypotheses or marginalize across them into the two general classes.

The utilities in Eqn. 3 can be converted back to probabilities via Bayesian decision theory $p_{\text {mod }}(H \mid D) \propto$ $\exp (\alpha U(H \mid D))$ (Frank \& Goodman, 2012) or any other decision-making algorithm. Within this paper, we will simply look at which hypothesis offers the highest utility, and leave it to future experimental work to model exactly how this is converted to belief strength. Loosely, then, a rational person believes in the conspiracy theory when $U\left(H_{C T} \mid D\right)$ is greater than $U\left(H_{N C} \mid D\right)$. When would this happen, and how does this connect to what we know about conspiratorial thinking?

\section{Four sources of rational conspiracies}

Based on the components of the utility calculation, there are at least four major ways that a CT can supplant the non-CT belief system within this rational framework. (We treat nonepistemic utilities as "rational" because the person is trying to solve the problem of which belief system brings the greatest utility to believe in, rather than which is the most correct, following Harris, Corner, and Hahn (2009) and others. If one considers these utilities to be irrational instead, the four ways drop to three, but the rational underpinnings remain.) 
Table 1: Disagreement from different priors. B is CTbeliever's probability, $\mathrm{N}$ is non-believer's. $H_{C T}$ is the $\mathrm{CT}$, $H_{N C}$ is the non-CT explanation.

\begin{tabular}{|cc|c|c|c|c|}
\hline & $\mathrm{H}$ & $p(H)$ & $p(D \mid H)$ & $U(H)$ & $U(H \mid D)$ \\
\hline $\mathrm{B}$ & $H_{C T}$ & 0.2 & 0.1 & 0 & $\mathbf{- 3 . 9 1}$ \\
\cline { 2 - 6 } & $H_{N C}$ & 0.8 & 0.9 & -4 & -4.33 \\
\hline \hline $\mathrm{N}$ & $H_{C T}$ & 0.05 & 0.1 & 0 & -5.30 \\
\cline { 2 - 6 } & $H_{N C}$ & 0.95 & 0.9 & -4 & $\mathbf{- 4 . 1 6}$ \\
\hline
\end{tabular}

We illustrate the way that each of these situations could lead to CT belief with sample calculations of Eqn. 3, shown in Tables 1-4. We will use the example of climate change denial to ground most of these analyses; we assume the average person thinks a CT has a low prior (5\%), low likelihood given the overwhelming scientific consensus (10\%), and finds moderate negative utility (-4) in believing that climate change is real (representing anxiety and actions they are undertaking to mitigate their footprint).

The top two rows of each table, labelled B, represent the relevant tweaks to the probability/utility values that would lead a person to prefer the CT explanation; the bottom rows, labelled $\mathrm{N}$, represent values the previously stated baseline values for non-CT belief. The specific numbers were chosen to illustrate the changes, and are not from experimentsthough they are partially based on values from Cook and Lewandowsky (2016)'s polarization experiments. Bold values show the maximum utility for the believer and nonbeliever, showing the different conclusions each reaches. Table 3 represents a different CT, discussed below.

Elevated priors Stronger beliefs in conspiracies in general-regardless of whether it is based in fact or a result of media consumption patterns that misinform-should raise a person's prior belief in CT explanations for new phenomena. This fits with one of the major findings about CT beliefs: that belief in one $\mathrm{CT}$ is correlated with belief in others, including fictitious CTs. Brotherton et al. (2013) show that CT beliefs appear to cluster, with the strongest influence on CT belief coming from structurally similar CTs (e.g., CTs involving extraterrestrials).

As shown in Table 1, even if two people agree on a given CT's likelihood and non-epistemic utility, the elevated CT prior may be enough to yield a higher posterior for a CT believer. In this example, the change from $5 \%$ to $20 \%$ prior expectation of a conspiracy is enough to convert the rational analyst $(\mathrm{N})$ into a $\mathrm{CT}$ believer $(\mathrm{B})$.

Different likelihood functions As in belief polarization, some people may find the data to be better explained by the $\mathrm{CT}$ than the non-CT explanation. Even if two people agree on the set of hypotheses, the observed data, and the conditional probability distributions that contribute to the $p(D \mid H)$ term, they may still end up with different values for $p(D \mid H)$ if they
Table 2: Disagreement from different likelihoods.

\begin{tabular}{|cc|c|c|c|c|}
\hline & $\mathrm{H}$ & $p(H)$ & $p(D \mid H)$ & $U(H)$ & $U(H \mid D)$ \\
\hline $\mathrm{B}$ & $H_{C T}$ & 0.05 & 0.5 & 0 & $\mathbf{- 3 . 6 9}$ \\
\cline { 2 - 6 } & $H_{N C}$ & 0.95 & 0.9 & -4 & -4.16 \\
\hline \hline \multirow{2}{*}{$\mathrm{N}$} & $H_{C T}$ & 0.05 & 0.1 & 0 & -5.30 \\
\cline { 2 - 6 } & $H_{N C}$ & 0.95 & 0.9 & -4 & $\mathbf{- 4 . 1 6}$ \\
\hline
\end{tabular}

Table 3: Disagreement from different hypothesis spaces. $H_{N C}$ is an implausible non-conspiratorial explanation; $H_{T}$ is the correct non-conspiratorial explanation.

\begin{tabular}{|cc|c|c|c|c|}
\hline & $\mathrm{H}$ & $p(H)$ & $p(D \mid H)$ & $U(H)$ & $U(H \mid D)$ \\
\hline $\mathrm{B}$ & $H_{C T}$ & 0.5 & 0.02 & 0 & $\mathbf{- 4 . 6 1}$ \\
\cline { 2 - 6 } & $H_{N C}$ & 0.5 & 0.01 & 0 & -5.30 \\
\hline \hline \multirow{2}{*}{$\mathrm{N}$} & $H_{C T}$ & 0.33 & 0.02 & 0 & -5.02 \\
\cline { 2 - 6 } & $H_{N C}$ & 0.33 & 0.01 & 0 & -5.71 \\
\cline { 2 - 6 } & $H_{T}$ & 0.33 & 0.75 & 0 & $\mathbf{- 1 . 4 0}$ \\
\hline
\end{tabular}

disagree on the value of another factor (Jern et al., 2014).

For instance, Cook and Lewandowsky (2016) show for climate change denial that a person's likelihood function for the scientific consensus of climate change depends on a third variable: whether the person trusts scientists or not. If the person trusts scientists not to lie, then the likelihood of the observed consensus is high if climate change is real and low if it is not. If the person believes scientists are likely to lie, the likelihood of the consensus is high if climate change is real and moderate if it is not. This can be enough to change the posteriors, as shown in Table 2; $p\left(D \mid H_{C T}\right)$ is higher for the CT believer, who believes scientists would lie to maintain a desired consensus, than the non-believer, who considers this unlikely.

Gaps in the hypothesis space The third potential source of accepting CT beliefs lies in cases where the true explanation is not part of the believer's hypothesis space. Some medical, scientific, and technological CTs work like this; the specific mechanism that generates the observed data is sufficiently complex or obscure that many people do not have a plausible explanation for the observed data.

The short-lived Wayfairgate CT is an example of this; someone noticed that Wayfair was selling cabinets at implausibly high prices, branded with a variety of rare girls' names, and many of these names appeared as the first name of girls who had been reported missing. For Wayfairgate believers, this was too much to be a coincidence, and they claimed this was evidence of online child trafficking. Nonbelievers did not accept this claim, but found it difficult to explain non-conspiratorially until journalists put together the full story. Wayfair released a statement explaining the cabinets' high prices, the database of missing children was not updated when children returned home, and the rare names all could be scraped from a list of names in the United States Social Security Names database (Kimball, 2020). Having the 
Table 4: Disagreement from different non-epistemic utilities.

\begin{tabular}{|cc|c|c|c|c|}
\hline & $\mathrm{H}$ & $p(H)$ & $p(D \mid H)$ & $U(H)$ & $U(H \mid D)$ \\
\hline \multirow{3}{*}{$\mathrm{B}$} & $H_{C T}$ & 0.05 & 0.1 & 3 & $\mathbf{- 2 . 3 0}$ \\
\cline { 2 - 6 } & $H_{N C}$ & 0.95 & 0.9 & -4 & -4.16 \\
\hline \hline $\mathrm{N}$ & $H_{C T}$ & 0.05 & 0.1 & 0 & -5.30 \\
\cline { 2 - 6 } & $H_{N C}$ & 0.95 & 0.9 & -4 & $\mathbf{- 4 . 1 6}$ \\
\hline
\end{tabular}

pieces to put together this true hypothesis $H_{T}$ required significant investigation, so many people were left with two notparticularly-compelling explanations, and CT believers never sought out the $H_{T}$ explanation. This situation is shown in Table 3, where the non-believer's hypothesis space includes a high-likelihood hypothesis that the CT-believer lacks.

Non-epistemic utilities The last source of CT beliefs in a rational model comes from enriching the notion of utility. Intuitively, the goal of a rational analysis of data and hypotheses is to determine which hypothesis "best" explains the data. Often, this is based strictly on epistemic utility, choosing the hypothesis with maximum posterior probability. But decision theory allows people make choices to maximize expected benefit, including non-epistemic utilities (Harris et al., 2009).

Such additional utilities/costs have been implemented in other rational frameworks to explain seemingly irrational choice behaviors. The Rational Speech Act model, for instance, assumes that speakers choose what to say in conversation based not only on how well it will help the listener understand the true state of the world (epistemic utility), but also on factors such as the social utility of a more positive statement. Such modifications of the utility function can explain the mild but polite untruth of a "white lie" (Yoon, Tessler, Goodman, \& Frank, 2016) or irony (Kao \& Goodman, 2015).

For CTs, there are many potential utilities to the CT (or costs to the non-CT) that could encourage the adoption of CT over a non-CT explanation. Gosa (2011) explains the prominence of CTs in hip-hop as a part of "counterknowledge", as well as a participatory "treasure hunt". Belief in CTs can yield a sense of community The non-epistemic utilities of a satisfying but implausible CT may be enough to override the greater epistemic utility of the more plausible non-CT account. In Table 4, the CT believer gains satisfaction from the counterknowledge or community of the CT that the nonbeliever does not.

Furthermore, the state of the world described by a CT is usually a worse world than the non-CT alternative. For instance, the Satanic panic, Pizzagate, and QAnon CTs posit huge child trafficking rings that do not exist in the non-CT alternative. Harris et al. (2009) showed that when people have both uncertainty about the exact probabilities of different states of the world and a perception that their choice may impact the outcome of an event, normative decision making with asymmetric loss functions will result in choices that appear to overestimate the probability of negative outcomes. If people believe that their belief in CTs can help avoid negative outcomes (e.g., by bringing the conspiracy to light before another conspiratorial action is committed), rational decision makers would be biased toward believing the $\mathrm{CT}$.

\section{Discussion}

Testing the predictions of this framework This framework is consistent with previous work that found behaviors around CTs and belief polarization show some signs of rationality. With the framework in place, we can directly test whether CT beliefs respond to probability and utility changes in a rational manner in future work.

Cook and Lewandowsky (2016)'s experiments on belief polarization can be adapted to the CT setting to assess whether changes in people's priors and likelihoods result in the anticipated changes to their posterior beliefs. As for the missing hypothesis case, participants can be given a hard-toexplain phenomenon, like a UFO sighting, and asked which of two vague explanations is more plausible, versus being given a complex but high-likelihood explanation. Utilitybased effects can be tested by asking about how satisfying or worrying different $\mathrm{CT}$ and non-CT explanations are.

The role of irrationality While this framework argues that belief in CTs can emerge from primarily rational frameworks, there is substantial evidence that CT belief is aided by nonnormativity in the reasoning process, such as confirmation biases, or environmental/personal factors like stress, anxiety, and loss of control. In laying out a rational framework, we are not discounting the role of non-normative factors, but rather seeking to show that CT belief can arise from rational analysis. It seems likely that CTs fall on a continuum from mostly rational to mostly irrational, and one goal of this framework is to help identify how responsive a given CT, or CT believer, is to rational analysis.

What can be done? The most important part of assessing the rational and irrational parts of CTs is to understand what interventions can help to avert dangerous CTs from taking hold in individuals and society. van der Linden et al. (2017) found that a joint affective/cognitive "inoculation" against misinformation reduced the influence of climate change CTs in their participants, consistent with the notion of CT belief being partially rational and partially irrational. Understanding the rational aspects of CTs may also help explain the correlation of CT belief with openness to new experiences (Swami et al., 2013). Lastly, the influence of priors suggests that building up trust to reduce overall belief in conspiracies may be an effective intervention against CTs.

\section{References}

Barkun, M. (2003). A Culture of Conspiracy: Apocalyptic Visions in Contemporary America. Berkeley: University of California Press. 
Benen, S. (2014, January). Reflecting on 'conspiracy theories'. https://www.msnbc.com/rachel-maddowshow/reflecting-conspiracy-theories-msna247541.

Brotherton, R., \& French, C. C. (2014). Belief in Conspiracy Theories and Susceptibility to the Conjunction Fallacy. Applied Cognitive Psychology, 28(2), 238-248.

Brotherton, R., French, C. C., \& Pickering, A. D. (2013). Measuring Belief in Conspiracy Theories: The Generic Conspiracist Beliefs Scale. Frontiers in Psychology, 4.

Cook, J., \& Lewandowsky, S. (2016). Rational Irrationality: Modeling Climate Change Belief Polarization Using Bayesian Networks. Topics in Cognitive Science, 8(1), 160-179.

Douglas, K. M., \& Sutton, R. M. (2008). The Hidden Impact of Conspiracy Theories: Perceived and Actual Influence of Theories Surrounding the Death of Princess Diana. The Journal of Social Psychology, 148(2), 210-222.

Frank, M. C., \& Goodman, N. D. (2012). Predicting pragmatic reasoning in language games. Science, 336, 998.

Franks, B., Bangerter, A., Bauer, M. W., Hall, M., \& Noort, M. C. (2017). Beyond "Monologicality"? Exploring Conspiracist Worldviews. Frontiers in Psychology, 8, 861.

Goreis, A., \& Voracek, M. (2019). A Systematic Review and Meta-Analysis of Psychological Research on Conspiracy Beliefs: Field Characteristics, Measurement Instruments, and Associations With Personality Traits. Frontiers in Psychology, 10, 205.

Gosa, T. L. (2011). Counterknowledge, racial paranoia, and the cultic milieu: Decoding hip hop conspiracy theory. Poetics, 39(3), 187-204.

Groh, D. (1987). The Temptation of Conspiracy Theory, or: Why Do Bad Things Happen to Good People? Part II: Case Studies. In C. F. Graumann \& S. Moscovici (Eds.), Changing Conceptions of Conspiracy (pp. 15-37). New York, NY: Springer New York.

Harris, A. J., Corner, A., \& Hahn, U. (2009). Estimating the probability of negative events. Cognition, 110(1), 51-64.

Hoyt, M. A., Rubin, L. R., Nemeroff, C. J., Lee, J., Huebner, D. M., \& Proeschold-Bell, R. J. (2012). HIV/AIDSrelated institutional mistrust among multiethnic men who have sex with men: Effects on HIV testing and risk behaviors. Health Psychology, 31(3), 269-277.

Huneman, P., \& Vorms, M. (2018). Is a Unified Account of Conspiracy Theories Possible? Argumenta Oeconomica Cracoviensia, 3, 49-72.

Jern, A., Chang, K.-m. K., \& Kemp, C. (2014). Belief polarization is not always irrational. Psychological Review, 121(2), 206-224.

Jolley, D., \& Douglas, K. M. (2014). The Effects of AntiVaccine Conspiracy Theories on Vaccination Intentions. PLoS ONE, 9(2), e89177.

Kao, J. T., \& Goodman, N. (2015). Let's talk (ironically) about the weather: Modeling verbal irony. Proceedings of the Cognitive Science conference.

Keeley, B. L. (1999). Of Conspiracy Theories. Journal of
Philosophy, 96.

Kimball, W. (2020, July). About the Wayfair Child Trafficking Conspiracy. https://gizmodo.com/about-the-wayfair-childtrafficking-conspiracy-1844342713.

Madsen, J. K., Bailey, R. M., \& Pilditch, T. D. (2018). Large networks of rational agents form persistent echo chambers. Scientific Reports, 8(1), 12391.

McCauley, C., \& Jacques, S. (1979). The popularity of conspiracy theories of presidential assassination: A Bayesian analysis. Journal of Personality and Social Psychology, 37(5), 637-644.

Navarro, D. J., \& Perfors, A. F. (2011). Hypothesis generation, sparse categories, and the positive test strategy. Psychological Review, 118(1), 120-134.

Olsson, E. J. (2020). Why Bayesian agents polarize. In F. Broncano-Berrocal \& J. A. Carter (Eds.), The Epistemology of Group Disagreement. Routledge.

Pagán, V. E. (2008). Toward a Model of Conspiracy Theory for Ancient Rome. New German Critique, 35(1), 27-49.

Raab, M. H., Ortlieb, S. A., Auer, N., Guthmann, K., \& Carbon, C.-C. (2013). Thirty shades of truth: Conspiracy theories as stories of individuation, not of pathological delusion. Frontiers in Psychology, 4.

Racioppi, D. (2016, November). Time to pay the toll: Two more guilty in Bridgegate scandal. https://www.northjersey.com/story/news/2016/11/04/timeto-pay-the-toll-two-more-guilty-in-bridgegatescandal/93445682/.

Swami, V. (2012). Social psychological origins of conspiracy theories: The case of the Jewish conspiracy theory in Malaysia. Frontiers in Psychology, 3, 280.

Swami, V., Pietschnig, J., Tran, U. S., Nader, I. W., Stieger, S., \& Voracek, M. (2013). Lunar lies: The impact of informational framing and individual differences in shaping conspiracist beliefs about the moon landings. Applied Cognitive Psychology, 27, 71-80.

Taber, C. S., \& Lodge, M. (2006). Motivated Skepticism in the Evaluation of Political Beliefs. American Journal of Political Science, 50(3), 755-769.

van der Linden, S., Leiserowitz, A., Rosenthal, S., \& Maibach, E. (2017). Inoculating the Public against Misinformation about Climate Change. Global Challenges, 1(2), 1600008.

Vul, E., Goodman, N., Griffiths, T. L., \& Tenenbaum, J. B. (2014). One and Done? Optimal Decisions From Very Few Samples. Cognitive Science, 38(4), 599-637.

Wood, M. J. (2016). Some Dare Call It Conspiracy: Labeling Something a Conspiracy Theory Does Not Reduce Belief in It. Political Psychology, 37(5), 695-705.

Wood, M. J., Douglas, K. M., \& Sutton, R. M. (2012). Dead and Alive: Beliefs in Contradictory Conspiracy Theories. Social Psychological and Personality Science, 3(6), 767773.

Yoon, E. J., Tessler, M. H., Goodman, N. D., \& Frank, M. C. (2016). Talking with tact: Polite language as a balance 
between kindness and informativity. Proceedings of the Cognitive Science conference.

Zimper, A., \& Ludwig, A. (2009). On attitude polarization under Bayesian learning with non-additive beliefs. Journal of Risk and Uncertainty, 39(2), 181-212. 